\title{
CONTEXT-BASED SEGMENTATION AND ANALYSIS OF MULTI-CYCLE REAL-TIME CARDIAC MRI
}

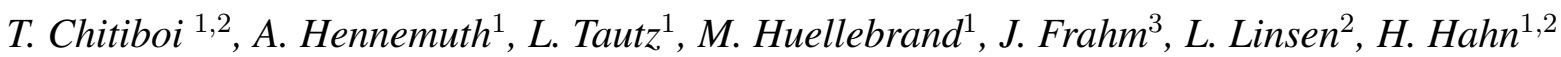 \\ ${ }^{1}$ Fraunhofer MEVIS Institute for Medical Image Computing, Bremen, Germany \\ 2 Jacobs University, Bremen, Germany \\ ${ }^{3}$ Max Planck Institute for Biophysical Chemistry, Göttingen, Germany
}

\begin{abstract}
The recent development of a real-time magnetic resonance imaging (MRI) technique with 20 to $30 \mathrm{~ms}$ temporal resolution allows for imaging multiple consecutive heart cycles, without the need for breath holding or ECG synchronization. Manual analysis of the resulting image series is no longer feasible because of their length. We propose a region-based algorithm for automatically segmenting the myocardium in consecutive heart cycles based on local context and prior knowledge. The method was evaluated on ten real-time MRI series and compared to segmentations by two observers, with promising results. We show that our approach enables a multicycle analysis of the heart function robust to breathing and arrhythmia.
\end{abstract}

Index Terms - myocardium, segmentation, cardiac function, real-time cardiac MRI.

\section{INTRODUCTION}

Cine MRI is a reference method for examining the left ventricle (LV) function, an important mean of diagnosing heart disease. With recent developments, real-time MRI examinations are possible without the need for breath holding and ECG synchronization. This new acquisition technique has numerous benefits: Apart from improving patient comfort, it reduces the artifacts caused by averaging several non-identical heart cycles. This allows to accurately examine patients with arrhythmia, which was unachievable using regular cine sequences.

Yet the problem of automatically segmenting the heart muscle (myocardium) is still open, since commercial solutions available for regular cine MRI are insufficiently reliable for medical practice. In this context, real-time images are even more challenging considering the length of the image series and the atypical T1 contrast properties which change during the heart cycle because of through-plane motion.

We propose a context-based automatic algorithm for segmenting the myocardium in short-axis real-time MR images. Our method uses prior knowledge integrated in a region-based graph representation of the image. This enables the segmentation of $2 \mathrm{D}$ temporal slices with the goal of visualizing the myocardium over time. Frames which are difficult to delineate independently can be solved by propagating results from neighboring time steps. Using this method it is possible to analyze and compare cardiac function over multiple heart cycles under various physiologic and pathological conditions.

\section{RELATED WORK}

Early attempts at real-time cardiac MRI by Finn et al. [1] were recently perfected by Uecker et al. [2] using highly undersampled radial FLASH with nonlinear inverse reconstruction (NLINV). Preliminary applications to cardiac MRI [3] achieved a high temporal resolution of 20 to $30 \mathrm{~ms}$ with an inplane resolution between 1.5 and $2 \mathrm{~mm}$. The potential of realtime MRI to reveal heart conditions previously undetectable with cine MRI has been discussed in detail in [4, 5].

A comprehensive survey of the work on automatic myocardium segmentation in short-axis cine-MRI was documented by Petitjean et al. [6]. The attempts cover a variety of segmentation methods. Pixel classification approaches that build a Gaussian mixture model on the image histogram [7] or use k-means clustering [8] are sensitive to noise, intensity variability due to different scanners and sequences, and turbulence in the blood pool. Active appearance models [9] have to deal with a high patient and image variability, and require a large amount of training data. Many approaches try to directly find the myocardial contours using deformable models on image gradient [10], gradient vector flow [11], or level sets [12]. However, these methods face difficulties for lowcontrast images and are susceptible to local minima or, on the contrary, find strongest borders that do not correspond to the myocardium. Other approaches use a combination of methods [13] or transform the image in polar coordinates [14], each having their own limitations regarding image contrast.

In conclusion, although there has been tremendous work towards an automatic segmentation of the myocardium, the problem has not yet been completely solved. Moreover, no specific attempts have hitherto been reported for the special conditions of a real-time MRI study. 


\section{OBJECT-BASED SEGMENTATION}

A major challenge of myocardium segmentation in real-time MRI is the low contrast between the heart muscle and surrounding structures such as the liver. Intensity variations over each heart cycle and the influence of breathing motion present further complications. In our approach we tackle these problems by making use of prior knowledge regarding the myocardium geometry and local image context such as the known spatial relations between different anatomical structures. The proposed method consists of three steps: detecting the heart center, object-based segmentation in $2 \mathrm{D}$, and contour propagation along the temporal domain.

First, a seed point $s$ is found close to the LV center in every frame. To account for the free breathing motion, the heart center is tracked over time using a Kalman filter. For a robust estimation of the LV center in the first frame, which is also the initial state of the Kalman filter, several temporal maximum intensity projections ranging between 10 and 100 time steps are individually analyzed using the Hough transform [15] to detect the most likely location of the LV circle.

In order to perform the object-based segmentation, images are initially partitioned into regions of about $50 \mathrm{~mm}^{2}$, using a $\mathrm{k}$-means clustering algorithm in three dimensions $(\mathrm{X}, \mathrm{Y}$, and intensity) called SLIC super-pixels [16]. The resulting regions (Fig.1-1) are stored in an attributed relational graph and managed using a generic framework introduced by Homeyer et al. [17]. The atomic regions, now called objects, are described by a set of properties regarding their intensity statistics, shape, and relation to other objects. The advantages of this image representation are the reduced sensitivity to noise of regions as opposed to individual pixels as well as the possibility to directly use local context from the relational graph to infer the myocardial border where contrast is low.

The LV blood pool is first estimated by region growing on the object level starting from $s$, based on an adaptive threshold. The threshold is iteratively updated using the median intensity of the resulting region and the process is repeated until the size and shape of the blood pool region falls within an expected range. Based on the known circular shape of the blood pool, a best-fitting ellipse is computed using principal component analysis (PCA). All neighboring objects covered more than $50 \%$ by the ellipse are merged, such that the dark papillary muscles are also included in the blood pool (Fig.1-2).

The lung is segmented using a threshold that separates the lowest intensity peak in the image histogram, followed by considering the largest connected component. The right ventricle (RV) is roughly estimated using the average intensity of the segmented LV as threshold. The correct connected component for the RV is identified by its size and its relative position to the $\mathrm{LV}$ and the lung. This method is successful in the middle and basal sections, as well as in the apical views at diastole where the RV blood pool is clearly visible. If the $\mathrm{RV}$ and lung are correctly identified, we can use their posi-
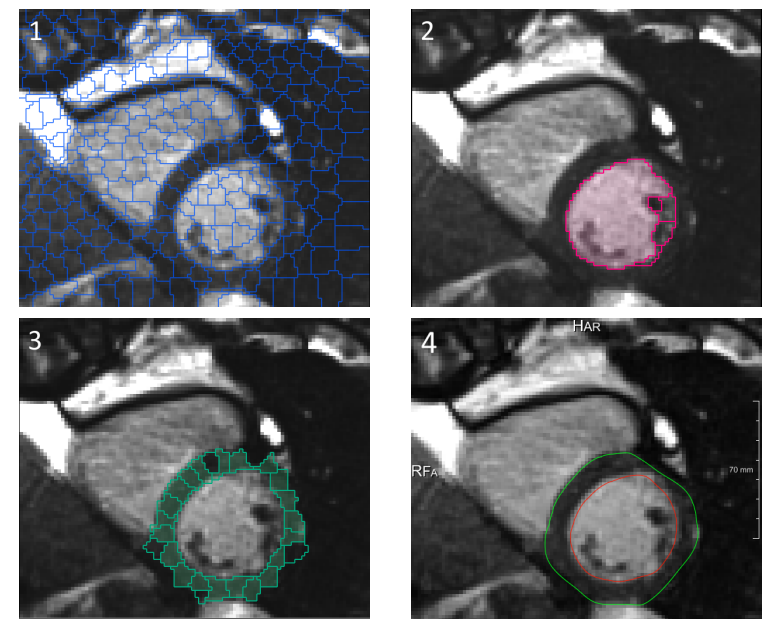

Fig. 1: (1) Initial objects; (2) Bloodpool objects; (3) Myocardium objects; (4) Result after active contour relaxation.

tion to separate the myocardium from the liver where contrast is poor. Objects falling bellow the line connecting the lower boundaries of the RV and lung are excluded from the myocardium, as shown in Fig.2 - left.

Next, the myocardium is initialized with the first ring of objects surrounding the blood pool. Further neighbors are added iteratively based on local similarity which tries to account for intensity non-uniformity in the heart muscle, as shown in Fig.1-3. Possible leakage into the nearby liver is counteracted by restricting the myocardium thickness. The expected width is computed per temporal slice according to the relative heart phase, assuming that the maximum wall thickness varies linearly between $7 \mathrm{~mm}$ at diastole and $30 \mathrm{~mm}$ at systole, and is inversely proportional to the size of the blood pool. The joint myocardium and blood pool mask is also processed by ellipse overlap and a minimal contour constraint, to optimize its shape. Finally, the coarse contour obtained on the object level is smoothed on a pixel level using morphological operations and an active contour relaxation (Fig.1-4). The object-based segmentation is described in more detail in [18].

However, some frames are more difficult to segment due to non-uniform intensity in the myocardium or low contrast. While the exact degree of accuracy achieved by the previous steps can only be assessed with human input, some obviously inaccurate contours can be pin-pointed by analyzing the temporal continuity of the image series. An outlier would have a poor overlap with its neighboring contours in the temporal domain or, considering the blood volume, violate the periodic ascending and descending curve of the heart cycles (Fig.4 top). Contours meeting any of these conditions are excluded and replaced by propagating the segmentation from correct neighboring time points (Fig.2 - right). This can be achieved using a general non-rigid registration method based on the Morphon algorithm, as proposed by Tautz et al. [19]. We have chosen this method because it is invariant to motion-induced intensity changes during the heart cycle. To ensure temporal 

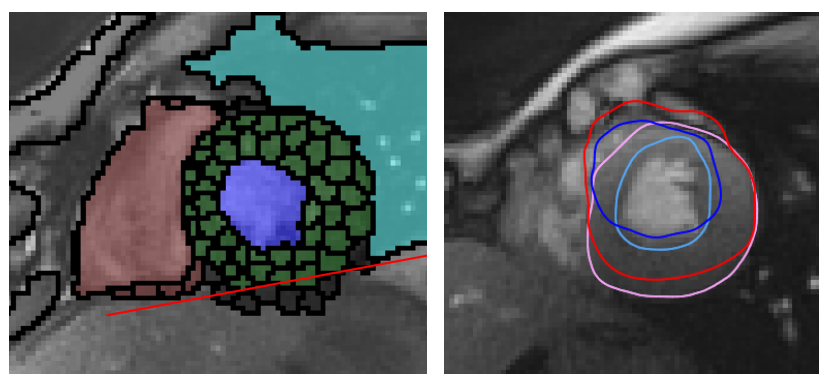

Fig. 2: Left: Image context - LV blood pool (blue), RV (red), lung (cyan), myocardium objects (green), and red line separating myocardium from liver. Right: Epicardial and endocardial contours after initial segmentation (red \& blue) and propagation from neighboring frames (pink \& cyan).

continuity over consecutively replaced contours, we interpolate between the results propagated from both directions.

\section{RESULTS AND DISCUSSION}

For the evaluation we have considered ten 2D real-time MRI series acquired from 4 different patients. Each sequence contains 10 to 15 cardiac cycles (350 to 500 time frames) acquired with a temporal resolution of $30 \mathrm{~ms}$. Each dataset was manually segmented by two different observers. The interobserver variability was quantified for the epicardium and endocardium (outer and inner myocardial contours) showing an average dice score of $96.3 \%$ and $94.2 \%$, respectively, and an average symmetrical distance of $1.24 \mathrm{~mm}$ and $1.34 \mathrm{~mm}$. The automatic segmentation results were compared against the two references. The dice score to the closest observer is depicted in Fig. 3 for each series. The average dice score compared to both observers was $95.1 \%$ for the epicardium and $89.8 \%$ for the endocardium, while the average distance lies at $1.48 \mathrm{~mm}$ and $1.93 \mathrm{~mm}$, respectively.

The segmentation accuracy for the epicardium is close to the inter-observer variability. However, in case of the endocardium some errors may still occur, such as the misclassification of papillary muscles and trabeculations. These structures are especially difficult to identify in the systolic (contraction) phase, when they become indistinguishable from the myocardium due to compression. This results in a general ten-

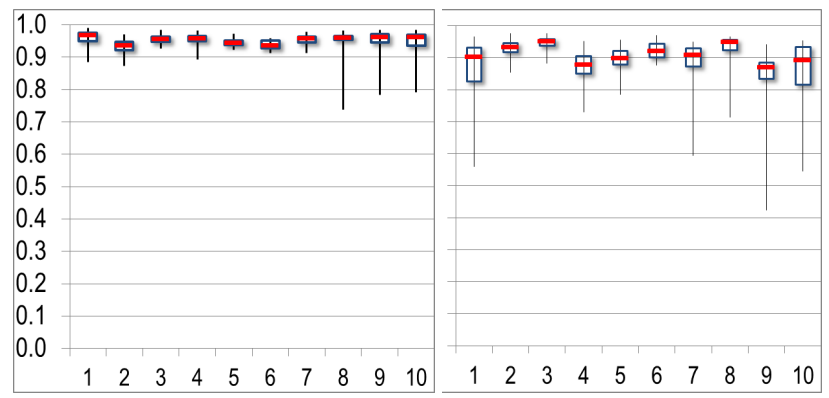

Fig. 3: (Left) epicardium and (right) endocardium dice score for the analysis of 10 real-time cardiac MRI series (short-axis view).
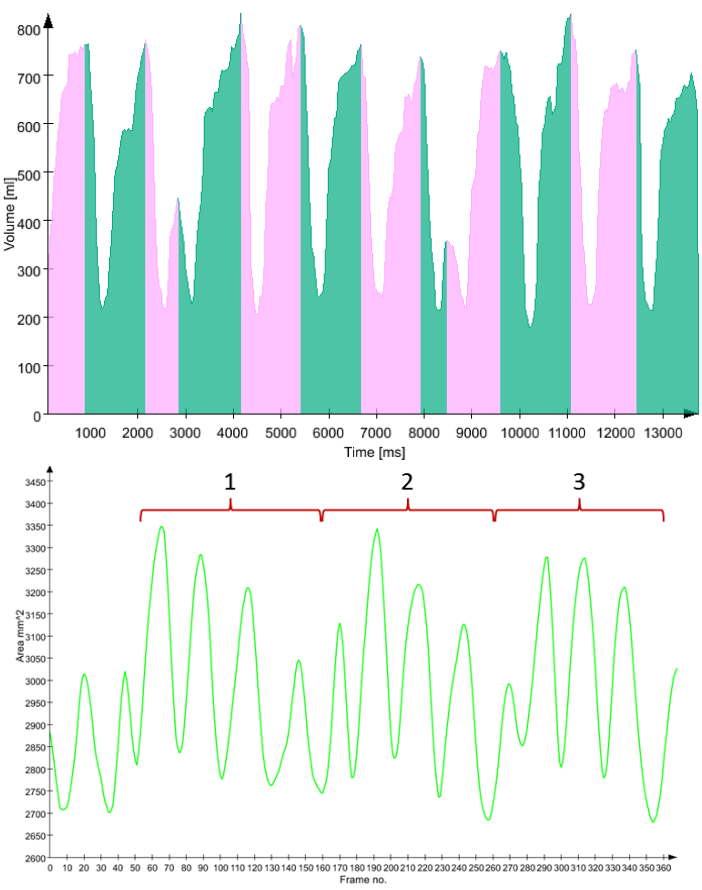

Fig. 4: (Top) Blood pool as a function of time for multiple heartbeats (mid-ventricular short-axis) for a patient with extrasystole due to arrhythmias. (Bottom) LV area as a function of time reveals the influence of breathing in a normal subject.

dency to underestimate the inner wall, which we counteract by performing a $3 \times 3$ morphological dilation in the detected systolic phase. Apical section (cases 9 and 10) remain the most difficult to segment, as can be observed in Fig.3. This is due to the more pronounced motion of the LV perpendicular to the image plane which causes sudden intensity variations and the loss of contrast between myocardium and blood pool.

\section{MULTI-CYCLE ANALYSIS}

The automatic segmentation of real-time cardiac MRI data in different short-axis views enables us to monitor the patient's heart function over several cardiac cycles, instead of considering only an averaged pseudo-cycle as captured by regular cine images. Fig.4 - top shows the segmented blood volume in a short-axis section as a function of time. The local minima and maxima are used to automatically determine the cardiac cycles. This analysis directly reveals the variability of heart function in patients with arrhythmia, where the blood volume per beat (stroke volume) or the ejection fraction can significantly differ from heartbeat to heartbeat.

Breathing may also influence heart function through pressure changes and the motion of the diaphragm. The heart volume (blood pool + myocardium) in a sample section can indeed be shown to vary periodically with the breathing cycle (Fig. 4 - bottom). Thus, our technique opens the possibility to analyze physiologic or pathologic variations of cardiac function for individual heartbeats and may offer new diagnostic 
opportunities by monitoring the responses to stress or exercise including Valsalva or Mueller maneuvers.

\section{CONCLUSION}

We have presented an automatic object-based segmentation algorithm for detecting the LV myocardium in real-time short-axis cardiac image series. Using this method, we could achieve a successful segmentation of the heart muscle in every frame and visualize the heart function over time. The approach is a prerequisite for a wider translation of real-time cardiac MRI into the clinical arena.

A current limitation is the restriction to individual 2D sections of the heart, because real-time images are acquired as serial cross-sectional views that cannot be directly stacked to form the left ventricle. However, future extensions will stitch the individual sections by matching similar cardiac cycles in order to form a $3 \mathrm{D}$ volumetric representation.

\section{REFERENCES}

[1] J. P. Finn, K. Nael, V. Deshpande, O. Ratib, and G. Laub, "Cardiac MR imaging: State of the technology," Radiology, vol. 241, no. 2, pp. 338-354, 2006.

[2] M. Uecker, S. Zhang, D. Voit, A. Karaus, K-D. Merboldt, and J. Frahm, "Real-time MRI at a resolution of 20 ms," NMR Biomed., vol. 23, no. 8, pp. 986-994, 2010 .

[3] S. Zhang, M. Uecker, D. Voit, K.D. Merboldt, and J. Frahm, "Real-time cardiovascular magnetic resonance at high temporal resolution: Radial flash with nonlinear inverse reconstruction," Cardiovasc. Magn. Reson., vol. 12, no. 39, 2010.

[4] C. Brinegar, Y-J. L. Wu, L. M. Foley, T. K. Hitchens, Q. Ye, C. Ho, and Z-P. Liang, "Real-time cardiac MRI without triggering, gating, or breath holding," EMBS, vol. 30, 2008.

[5] M. Francone, S. Dymarkowski, M. Kalantzi, and J. Bogaert, "Real-time cine MRI of ventricular septal motion: A novel approach to assess ventricular coupling," J. Magn. Reson. Imaging, vol. 21, no. 3, pp. 305-309, Mar 2005.

[6] C. Petitjean and J-N. Dacher, "A review of segmentation methods in short axis cardiac MR images," Med. Image Anal., vol. 15, no. 2, pp. 169-184, 2011.

[7] U. Kurkure, A. Pednekar, R. Muthupillai, S. D. Flamm, and I. A. Kakadiaris, "Localization and segmentation of left ventricle in cardiac cine-MR images," IEEE Trans. Biomed. Eng., vol. 56, no. 5, pp. 1360-1370, 2009.
[8] M. Lynch, O. Ghita, and P. F. Whelan, "Automatic segmentation of the left ventricle cavity and myocardium in MRI data," Comput. Biol. Med., vol. 36, no. 4, pp. 389-407, 2006.

[9] M. Üzümcü, A. Frangi, M. Sonka, J. Reiber, and B. Lelieveldt, "ICA vs. PCA active appearance models: Application to cardiac mr segmentation," MICCAI, pp. 451-458, 2003.

[10] A. Gupta, L. Von Kurowski, A. Singh, D. Geiger, CC. Liang, M-Y. Chiu, LP. Adler, M. Haacke, and DL. Wilson, "Cardiac MR image segmentation using deformable models," in Proc. Computers in Cardiology. IEEE, 1993, pp. 747-750.

[11] MF. Santarelli, V. Positano, C. Michelassi, M. Lombardi, L. Landini, et al., "Automated cardiac MR image segmentation: theory and measurement evaluation.," Med. Eng. \& Phys., vol. 25, no. 2, pp. 149, 2003.

[12] M. Lynch, O. Ghita, and P. F. Whelan, "Segmentation of the left ventricle of the heart in 3-D+ $t$ MRI data using an optimized nonrigid temporal model," IEEE Trans. Med. Imag., vol. 27, no. 2, pp. 195-203, 2008.

[13] M-P. Jolly, "Automatic segmentation of the left ventricle in cardiac MR and CT images," IJCV, vol. 70, no. 2, pp. 151-163, 2006.

[14] H-Y. Lee, N. CF. Codella, M. D. Cham, J. W. Weinsaft, and Y. Wang, "Automatic left ventricle segmentation using iterative thresholding and an active contour model with adaptation on short-axis cardiac MRI," IEEE Trans. Biomed. Eng., vol. 57, no. 4, pp. 905-913, 2010.

[15] R.K.K. Yip, P.K.S. Tam, and D.N.K. Leung, "Modification of hough transform for circles and ellipses detection using a 2-dimensional array," Pattern Rec., vol. 25, no. 9, pp. 1007-1022, 1992.

[16] R. Achanta, A. Shaji, K. Smith, A. Lucchi, P. Fua, and S. Süsstrunk, "Slic superpixels," École Polytechnique Fédéral de Laussanne, Tech. Rep, vol. 149300, 2010.

[17] A. Homeyer, M. Schwier, and HK Hahn, "A Generic Concept for Object-based Image Analysis," in VISAPP, 2010, vol. 2, pp. 530-533.

[18] T. Chitiboi, A. Hennemuth, L. Linsen, and HK. Hahn, "Towards automatic extraction of the myocardium in temporal MRI using object-based segmentation," in VMLS. The Eurographics Association, 2013, pp. 13-17.

[19] L. Tautz, A. Hennemuth, M. Andersson, A. Seeger, H. Knutsson, and O. Friman, "Phase-based Non-rigid Registration of Myocardial Perfusion MR Image Sequences," in IEEE ISBI, 2010, pp. 516 - 519. 